\title{
Chest X-ray for predicting mortality and the need for ventilatory support in COVID-19 patients presenting to the emergency department
}

\author{
Maurizio Balbi ${ }^{1,2} \mathbb{D} \cdot$ Anna Caroli $^{3} \cdot$ Andrea Corsi $^{1,2} \cdot$ Gianluca Milanese $^{4} \cdot$ Alessandra Surace $^{1,2} \cdot$ Fabiano Di Marco $^{5,6}$. \\ Luca Novelli ${ }^{5}$. Mario Silva ${ }^{4}$. Ferdinando Luca Lorini ${ }^{7}$. Andrea Duca ${ }^{8} \cdot$ Roberto Cosentini $^{8} \cdot$ Nicola Sverzellati $^{4}$. \\ Pietro Andrea Bonaffini ${ }^{1,2} \cdot$ Sandro Sironi ${ }^{1,2}$
}

Received: 5 June 2020 / Revised: 1 September 2020 / Accepted: 8 September 2020 / Published online: 8 October 2020

(C) The Author(s) 2020

\begin{abstract}
Objectives To evaluate the inter-rater agreement of chest X-ray (CXR) findings in coronavirus disease 2019 (COVID-19) and to determine the value of initial CXR along with demographic, clinical, and laboratory data at emergency department (ED) presentation for predicting mortality and the need for ventilatory support.

Methods A total of 340 COVID-19 patients who underwent CXR in the ED setting (March 1-13, 2020) were retrospectively included. Two reviewers independently assessed CXR abnormalities, including ground-glass opacities (GGOs) and consolidation. Two scoring systems (Brixia score and percentage of lung involvement) were applied. Inter-rater agreement was assessed by weighted Cohen's kappa $(\kappa)$ or intraclass correlation coefficient (ICC). Predictors of death and respiratory support were identified by logistic or Poisson regression.

Results GGO admixed with consolidation $(n=235,69 \%)$ was the most common CXR finding. The inter-rater agreement was almost perfect for type of parenchymal opacity $(\kappa=0.90)$, Brixia score $(\mathrm{ICC}=0.91)$, and percentage of lung involvement $(\mathrm{ICC}=$ 0.95). The Brixia score (OR: $1.19 ; 95 \% \mathrm{CI}: 1.06,1.34 ; p=0.003)$, age (OR: $1.16 ; 95 \% \mathrm{CI}: 1.11,1.22 ; p<0.001), \mathrm{PaO}_{2} / \mathrm{FiO}_{2} \mathrm{ratio}$ (OR: $0.99 ; 95 \%$ CI: $0.98,1 ; p=0.002$ ), and cardiovascular diseases (OR: 3.21; 95\% CI: 1.28, 8.39; $p=0.014$ ) predicted death. Percentage of lung involvement (OR: $1.02 ; 95 \%$ CI: $1.01,1.03 ; p=0.001)$ and $\mathrm{PaO}_{2} / \mathrm{FiO}_{2}$ ratio (OR: 0.99; 95\% CI: 0.99, 1.00; $p<0.001)$ were significant predictors of the need for ventilatory support.

Conclusions CXR is a reproducible tool for assessing COVID-19 and integrates with patient history, $\mathrm{PaO}_{2} / \mathrm{FiO}_{2}$ ratio, and $\mathrm{SpO} 2$ values to early predict mortality and the need for ventilatory support.

Key Points

- Chest X-ray is a reproducible tool for assessing COVID-19 pneumonia.

- The Brixia score and percentage of lung involvement on chest X-ray integrate with patient history, $\mathrm{PaO}_{2} / \mathrm{FIO}_{2}$ ratio, and $\mathrm{SpO}_{2}$ values to early predict mortality and the need for ventilatory support in COVID-19 patients presenting to the emergency department.
\end{abstract}

Electronic supplementary material The online version of this article (https://doi.org/10.1007/s00330-020-07270-1) contains supplementary material, which is available to authorized users.

Maurizio Balbi

balbi.m@libero.it

1 Department of Radiology, Azienda Socio-Sanitaria Territoriale Papa Giovanni XXIII, Bergamo, Italy

2 University of Milano-Bicocca, Milan, Italy

3 Bioengineering Department, Istituto di Ricerche Farmacologiche Mario Negri IRCCS, Bergamo, Italy
4 Scienze Radiologiche, Department of Medicine and Surgery, University of Parma, Parma, Italy

5 Respiratory Unit, Azienda Socio-Sanitaria Territoriale Papa Giovanni XXIII, Bergamo, Italy

6 Department of Health Sciences, University of Milan, Milan, Italy

7 Department of Anesthesia and Intensive Care, Azienda Socio-Sanitaria Territoriale Papa Giovanni XXIII, Bergamo, Italy

8 Emergency Department (Emergenza Alta Specializzazione), Azienda Socio-Sanitaria Territoriale Papa Giovanni XXIII, Bergamo, Italy 
Keywords Radiography · COVID-19 · Severe acute respiratory syndrome coronavirus 2

\begin{tabular}{|c|c|}
\hline Abbreviations & \\
\hline COVID-19 & Coronavirus disease 2019 \\
\hline CXR & Chest radiography \\
\hline ED & Emergency department \\
\hline $\mathrm{FiO}_{2}$ & Fraction of inspired oxygen \\
\hline GGO & Ground-glass opacity \\
\hline ICC & Intraclass correlation coefficient \\
\hline IQR & Interquartile range \\
\hline $\mathrm{PaO}_{2}$ & Arterial partial pressure of oxygen \\
\hline RT-PCR & $\begin{array}{l}\text { Real-time reverse transcriptase- } \\
\text { polymerase chain reaction }\end{array}$ \\
\hline SARS-CoV-2 & $\begin{array}{l}\text { Severe acute respiratory syndrome } \\
\text { coronavirus } 2\end{array}$ \\
\hline $\mathrm{SpO}_{2}$ & Oxygen saturation \\
\hline
\end{tabular}

\section{Introduction}

Coronavirus disease 2019 (COVID-19) has been declared a pandemic emergency by the World Health Organization on March 11, 2020 [1]. This infectious disease can result in a range of clinical outcomes, from an asymptomatic or mild flu-like illness to severe pneumonia, multiorgan failure, and even death [2]. The diagnosis of COVID-19 is based on the detection of the severe acute respiratory syndrome coronavirus 2 (SARS-CoV-2) by real-time reverse transcriptase-polymerase chain reaction (RT-PCR) testing, most commonly of a nasopharyngeal swab [3]. However, this method has some limitations: it is not universally available, turnaround times can be lengthy, and reported sensitivities vary $(30-70 \%)[4,5]$.

In the context of the COVID-19 pandemic, imaging has turned out to be a valuable complementary tool to "rule-in" or "rule out" suspected COVID-19 patients, potentially accelerating the speed of diagnosis compared with RT-PCR dynamics [6]. The choice of whether to use chest radiography (CXR) or computed tomography $(\mathrm{CT})$ as a first-line imaging modality for the assessment of COVID-19 depends on factors that vary considerably among scenarios (e.g., local resources, expertise) [7]. In Europe, as well as in the USA, CXR has been extensively used to triage patients with clinical suspicion of COVID-19 [8,9]. Even though CXR is less sensitive than CT, especially in the early stage of the disease, it is widely available and relatively inexpensive, can be performed at the bedside, and allows relative rapid cleaning and turn-over between patients, thus minimizing the risk of cross-link infection [10].
The spectrum of chest imaging manifestations of COVID19 on CXR has been extensively described [11, 12]. However, while the utility of CXR in predicting clinical outcomes has been investigated in the severe acute respiratory syndrome (SARS) coronavirus as well as in a variety of other types of pneumonia $[13,14]$, very few studies have assessed the prognostic value of CXR in COVID-19 patients $[15,16]$. Moreover, data about the reproducibility of CXR findings in COVID-19 still lack.

Therefore, our study aimed (a) to evaluate the inter-rater agreement of initial CXR findings in COVID-19 patients presenting to the emergency department (ED) during the early stage of the pandemic and (b) to determine the value of initial CXR findings combined with demographic, clinical, and laboratory data at ED presentation for predicting mortality and the need for ventilatory support in COVID19 patients.

\section{Materials and methods}

The Institutional Review Board (Comitato Etico di Bergamo, Italy) approved this retrospective observational study and waived the written informed consent.

\section{Study population}

A total of 359 consecutive patients presenting to the EDs of two affiliated hospitals (Papa Giovanni XXIII and San Giovanni Bianco, Bergamo, Italy) between March 1 and 13, 2020, were considered eligible for inclusion. The inclusion criteria were the following: (a) initial CXR performed in the ED setting and (b) final diagnosis of COVID-19 confirmed by a positive RT-PCR test. The exclusion criteria were (a) unavailable clinical or laboratory data $(n=5)$ and (b) non-diagnostic CXR image quality $(n=$ 14). Finally, a total of 340 patients were retrospectively enrolled.

\section{Data collection}

Demographic, clinical, and laboratory data were collected from patients' medical records. The recorded data included the following: age, sex, medical comorbidities, symptoms, clinical and laboratory data within $24 \mathrm{~h}$ of ED presentation (including the oxygen saturation $\left[\mathrm{SpO}_{2}\right]$, fraction of inspired oxygen $\left[\mathrm{FiO}_{2}\right]$, arterial partial pressure 
of oxygen $\left[\mathrm{PaO}_{2}\right]$, and $\mathrm{PaO}_{2} / \mathrm{FiO}_{2}$ ratio), and mode of respiratory support (oxygen mask, continuous positive airway pressure/noninvasive mechanical ventilation, invasive mechanical ventilation). For patients admitted to the intensive care unit, the highest levels of positive endexpiratory pressure, the use of extracorporeal membrane oxygenation, and prone positioning were also recorded. Patient length of stay was calculated by subtracting the date of ED presentation from the date of discharge or death. Patient survival status, as well as the date of death, was obtained from the Regional Healthcare Information System (SISS, Regione Lombardia, Italy) as of May 12, 2020.

\section{Imaging acquisition and analysis}

Images were acquired using digital radiographic systems (Definium 8000, GE Healthcare; FDR AcSelerate, Fujifilm Corporation) with tube voltages ranging from 120 to $150 \mathrm{kVp}$ and by employing automatic exposure control. The imaging data included CXR images acquired in the posteroanterior and lateral (PA/LAT, $n=130)$ or anteroposterior (AP, $n=210$ ) projections. The latter was performed when the patient was too unwell to tolerate standing. Only the AP and PA images were selected and retrospectively evaluated by two reviewers (G.M., a thoracic radiologist with 5 years of experience in a referral center; M.B., a fourth-year radiology resident), blinded to patient history other than COVID-19 positivity. Reviewers independently assessed the presence of lung abnormalities, including ground-glass opacities (GGOs), consolidation, and pulmonary nodules [17]. Distribution of GGOs and consolidation was classified as follows: (a) peripheral (involving mainly the peripheral one-third of the lung), central (involving mainly the central twothirds of the lung), or neither; (b) unilateral or bilateral; (c) upper zone (above the inferior wall of the aortic arch), middle zone (between the inferior wall of the aortic arch and the right inferior pulmonary vein), lower zone (below the right inferior pulmonary vein), or no zonal predominance. The presence of pleural effusion was assessed. The two reviewers were also asked to grade each CXR using the Brixia scoring system, an experimental 18-points severity scoring system designed for the assessment of COVID-19 pneumonia [18]. The Brixia score is obtained by dividing each lung into 3 zones (upper, middle, and lower zone, as explained above) and then scoring each zone from 0 to 3 based on types of pulmonary infiltrates detected, as follows: 0 , no lung abnormalities; 1 , interstitial infiltrates; 2, interstitial and alveolar infiltrates (interstitial predominance); 3, interstitial and alveolar infiltrates (alveolar predominance). The terms "interstitial infiltrate" and "alveolar infiltrate" used in the Brixia scoring system were reported in the current study as GGO and consolidation, respectively. CXRs showing only abnormalities other than GGOs and consolidation were scored as 0 . Pure consolidation was scored as 3 . The number of zones involved was also recorded. In addition, the overall extent of GGOs and consolidation was assessed by visually estimating and then averaging the percentage of involvement within each lung.

\section{Statistical analysis}

Patient data and CXR findings were reported as median and interquartile range (IQR) in case of continuous variables, or numbers and frequency distribution (\%) in case of binary or categorical variables.

CXR findings' inter-rater agreement was assessed both in the whole group and in subgroups with AP and PA radiographs by weighted Cohen's kappa (categorical variables), or intraclass correlation coefficient (ICC) (quantitative variables, namely the number of lung zones involved, Brixia score, and percentage of lung involvement). Moreover, the agreement in Brixia score and percentage of lung involvement was visualized by correlation and Bland-Altman plots.

Predictors of death and mode of respiratory support were identified among age, sex, comorbidities, duration of symptoms, $\mathrm{SpO}_{2}$, and $\mathrm{PaO}_{2} / \mathrm{FiO}_{2}$ ratio, as well as CXR findings (laterality, type of parenchymal opacity, number of lung zones involved, Brixia score, and percentage of lung involvement), by logistic and ordinal logistic regression, respectively. In all cases, univariate analyses were first performed to identify possible predictors. All variables with significant contributions at univariate analysis were included in the multivariate analysis, and main predictors were finally identified by reducing the multivariate model using a stepwise model selection technique. CXR findings refer to the most experienced reviewer, and only patients with no missing data were included in the regression analyses.

Significance of the differences in demographic, clinical, and laboratory data between patients with mild (Brixia score $<8$ ) and severe (Brixia score $\geq 8$ ) CXR findings was assessed by two-tail independent $t$ test (continuous variables) or chi-squared test (binary and categorical variables). Significance of the differences in the demographic, clinical, laboratory, and radiological features between deceased and survived patients was assessed by the Mann- 
Whitney test (numerical variables) or chi-squared test (binary and categorical variables).

Survival curves and pertinent $95 \%$ confidence intervals were computed using the Kaplan-Meier method for the whole patient cohort as well as for patients grouped by individual grouping variables (age, sex, number of comorbidities, $\mathrm{PaO}_{2} /$ $\mathrm{FiO}_{2}$ ratio, CXR findings at ED presentation). The significance of the difference between strata was computed by logrank test. The distribution by the most invasive respiratory support employed of age, $\mathrm{PaO}_{2} / \mathrm{FiO}_{2}$ ratio, Brixia score, percentage of lung involvement, and number of lung zones involved was displayed by boxplots.

In all tests, statistical significance was set at $p<0.05$. All statistical analyses were performed using R software, version 3.6.3.

\section{Results}

The main demographic, clinical, and laboratory features at ED presentation of the 340 COVID-19 patients included in the study are listed in Table 1 . Most patients were male $(252 / 340,74 \%)$. The median age was 68 (IQR $=57-76)$. Arterial hypertension represented the most common comorbidity $(162 / 340,48 \%)$, followed by cardiovascular diseases $(86 / 340,25 \%)$. The median number of days from symptom onset to ED presentation was 7 , with the most common symptoms being fever $(296 / 340,87 \%)$, dyspnea $(224 / 340,66 \%)$, and cough $(167 / 340,49 \%)$. The main blood test alterations were lymphocytopenia (131/190, $69 \%$ ), increased levels of C-reactive protein (323/333; $97 \%)$, lactate dehydrogenase $(278 / 306,91 \%)$, and aspartate transaminase $(215 / 331,65 \%)$. The median $\mathrm{PaO}_{2} / \mathrm{FiO}_{2}$ ratio was 238 (IQR $=143-285)$.

All patients underwent CXR and RT-PCR testing within the first $24 \mathrm{~h}$ of ED presentation. Initial RT-PCR tests were performed using nasopharyngeal swabs, according to the protocol established by the World Health Organization [3]. A total of 313 (92\%) enrolled patients had a positive initial RT-PCR test result, while 27 had a negative one. The latter were found to have a positive result at a second $(n=20)$ or third ( $n=5)$ RT-PCR test from a nasopharyngeal swab, up to a maximum of 8 days after ED referral. Only in 2 cases, the diagnosis of COVID-19 was confirmed by a positive RT-PCR test from bronchoalveolar lavage fluid performed 4 and 14 days after the ED presentation, respectively. All of the 27 patients who tested negative on initial RT-PCR had CXR findings suggestive of pneumonia, while 6 patients were negative for both reviewers on CXR and tested positive on initial RT-PCR. There were no statistically significant differences in CXR findings between patients with
Table 1 Summary of data obtained within $24 \mathrm{~h}$ of ED presentation in 340 patients with confirmed COVID-19

\begin{tabular}{|c|c|}
\hline Total no. & 340 \\
\hline RT-PCR, positive initial results/total no. (\%) & $313 / 340(92 \%)$ \\
\hline \multicolumn{2}{|l|}{ Age } \\
\hline Median [IQR], year & $68[57-76]$ \\
\hline \multicolumn{2}{|l|}{ Distribution, no./total no. (\%) } \\
\hline $18-59$ year & $101 / 340(30 \%)$ \\
\hline $60-69$ year & $86 / 340(25 \%)$ \\
\hline 70-79 year & $96 / 340(28 \%)$ \\
\hline$\geq 80$ year & $57 / 340(17 \%)$ \\
\hline Gender, F, no./total no. (\%) & $88 / 340(26 \%)$ \\
\hline \multicolumn{2}{|l|}{ Smoking history, no./total no. (\%) } \\
\hline Never smoked & $102 / 165(62 \%)$ \\
\hline Former smoker & $54 / 165(33 \%)$ \\
\hline Current smoker & $9 / 165(5 \%)$ \\
\hline \multicolumn{2}{|l|}{ Comorbidities, no./total no. (\%) } \\
\hline Any & $167 / 215(78 \%)$ \\
\hline$>2$ & $57 / 215(27 \%)$ \\
\hline Arterial hypertension & $162 / 340(48 \%)$ \\
\hline Cardiovascular disease ${ }^{* *}$ & $86 / 340(25 \%)$ \\
\hline Obesity*** & $50 / 215(23 \%)$ \\
\hline Diabetes & $54 / 340(16 \%)$ \\
\hline Dyslipidemia & $28 / 340(8 \%)$ \\
\hline COPD & $22 / 340(6 \%)$ \\
\hline Chronic renal failure & $12 / 340(4 \%)$ \\
\hline Neoplasia (active history) & $26 / 340(8 \%)$ \\
\hline Rheumatic pathology & $18 / 340(5 \%)$ \\
\hline Immunodepression & $20 / 340(6 \%)$ \\
\hline Epilepsy & $3 / 340(1 \%)$ \\
\hline Cirrhosis & $6 / 340(2 \%)$ \\
\hline \multicolumn{2}{|l|}{ Symptoms, no./total no. (\%) } \\
\hline Fever & $296 / 340(87 \%)$ \\
\hline Cough & $167 / 340(49 \%)$ \\
\hline Dyspnea & $224 / 340(66 \%)$ \\
\hline Pharyngodynia & $9 / 340(3 \%)$ \\
\hline Asthenia & $77 / 340(23 \%)$ \\
\hline Anorexia & $18 / 340(5 \%)$ \\
\hline Myalgia & $12 / 340(4 \%)$ \\
\hline Diarrhea & $19 / 340(6 \%)$ \\
\hline Nausea & $15 / 340(4 \%)$ \\
\hline Vomit & $16 / 340(5 \%)$ \\
\hline Dizziness & $18 / 340(5 \%)$ \\
\hline Abdominal pain & $6 / 340(2 \%)$ \\
\hline Chest pain & $12 / 340(4 \%)$ \\
\hline Duration of symptoms, no. with data & 332 \\
\hline Median [IQR], days & $7[5-10]$ \\
\hline \multicolumn{2}{|l|}{ Laboratory data } \\
\hline $\mathrm{SpO}_{2} *$, no. with data & 277 \\
\hline Median [IQR], \% & $90[86-94]$ \\
\hline
\end{tabular}


Table 1 (continued)

$\mathrm{PaO}_{2} / \mathrm{FiO}_{2}$ ratio, no. with data Median [IQR]

Distribution, no./total no.

$<100$, severe ARDS

100-200, moderate ARDS

200-300, mild ARDS

$>300$, normal

$\mathrm{HB}, \mathrm{g} / \mathrm{dL}$

Median [IQR]

$<14$, no./total no. $(\%)$

$>17$, no./total no. $(\%)$

$\mathrm{WBC}, / \mathrm{mm}^{3}$

Median [IQR]

$<4000$, no./total no. (\%)

$>10000$, no./total no. (\%)

Neutrophils

Median [IQR] (WBC \%)

Median [IQR] $\left(/ \mathrm{mm}^{3}\right)$

$<2000$, no./total no. (\%)

$>6700$, no./total no. (\%)

Lymphocytes

Median [IQR] (WBC \%)

Median [IQR] $\left(/ \mathrm{mm}^{3}\right)$

$<1000$, no./total no. $(\%)$

Monocytes

Median [IQR] (WBC \%)

Median [IQR] $\left(/ \mathrm{mm}^{3}\right)$

$<250$, no./total no. (\%)

$>800$, no./total no. $(\%)$

Eosinophils

Median [IQR] (WBC \%)

Median [IQR] $\left(/ \mathrm{mm}^{3}\right)$

$>500$, no./total no. (\%)

Basophils

Median [IQR] (WBC \%)

Median [IQR] $\left(/ \mathrm{mm}^{3}\right)$

$>100$, no./total no. $(\%)$

PLT, $/ \mathrm{mm}^{3}$

Median [IQR]

$<150,000$, no./total no. $(\%)$

$>400,000$, no./total no. (\%)

INR

Median [IQR]

$>1.25$, no./total no. $(\%)$

aPTT ratio

Median [IQR]

$>1.25$, no./total no. $(\%)$

AST, U/L

Median [IQR]

$>40$, no./total no. $(\%)$

ALT, U/L

Median [IQR]

$>40$, no./total no. (\%)

Creatinine, $\mathrm{mg} / \mathrm{dL}$

Median [IQR]

$>1.30$, no./total no. (\%)

Urea, $\mathrm{mg} / \mathrm{dL}$

Median [IQR]

$>50$, no./total no. $(\%)$

LDH, U/L

Median [IQR]

$\geq 250$, no./total no. (\%)
258

238 [143-285]

$41 / 258(16 \%)$

$60 / 258(23 \%)$

$113 / 258(44 \%)$

$44 / 258(17 \%)$

13.8 [12.5-14.9]

$182 / 338(54 \%)$

$10 / 338(3 \%)$

6380 [4865-9412]

$45 / 338(13 \%)$

$66 / 338(20 \%)$

78 [72-86]

4999 [3428-7665]

$16 / 287(6 \%)$

$89 / 287(31 \%)$

13 [8-18]

805 [570-1088]

$131 / 190(69 \%)$

6[4-8]

362 [245-554]

$50 / 190(26 \%)$

$16 / 190(8 \%)$

$0[0-0.3]$

0 [0-14.2]

$2 / 190(1 \%)$

$0.2[0.1-0.3]$

$11.9[8.7-23.7]$

$3 / 190(2 \%)$

$177,000[140,000-226,000]$

$98 / 321(31 \%)$

$8 / 321(2 \%)$

1.07 [1.02-1.15]

$34 / 279(12 \%)$

1.13 [1.02-1.26]

$86 / 306(28 \%)$

52 [37-77]

215/331 (65\%)

39 [26-60]

$149 / 335(44 \%)$

0.93 [0.77-1.23]

$76 / 337(23 \%)$

$43[33-65]$

$109 / 285(38 \%)$

404 [314-551]

$278 / 306(91 \%)$
Table 1 (continued)

\begin{tabular}{|c|c|}
\hline \multicolumn{2}{|l|}{$\mathrm{CRP}, \mathrm{mg} / \mathrm{dL}$} \\
\hline Median [IQR] & 12 [6-18] \\
\hline$\geq 1$, no./total no. $(\%)$ & $323 / 333(97 \%)$ \\
\hline \multicolumn{2}{|l|}{ Fibrinogen, $\mathrm{g} / \mathrm{dL}$} \\
\hline Median [IQR] & $0.63[0.52-0.73]$ \\
\hline$<0.150$, no. $/$ total no. $(\%)$ & $0 / 63(0 \%)$ \\
\hline$>0.400$, no./total no. $(\%)$ & $59 / 63(94 \%)$ \\
\hline \multicolumn{2}{|l|}{$\mathrm{Na}, \mathrm{mEq} / \mathrm{L}$} \\
\hline Median [IQR] & 138 [136-140] \\
\hline$<136$, no./total no. $(\%)$ & $78 / 335(23 \%)$ \\
\hline$>145$, no./total no. $(\%)$ & $9 / 335(3 \%)$ \\
\hline \multicolumn{2}{|l|}{$\mathrm{K}, \mathrm{mEq} / \mathrm{L}$} \\
\hline Median [IQR] & $3.9[3.6-4.3]$ \\
\hline$<3.5$, no./total no. $(\%)$ & $55 / 331(17 \%)$ \\
\hline$>5$, no./total no. $(\%)$ & $19 / 331(6 \%)$ \\
\hline \multicolumn{2}{|l|}{$\mathrm{Cl}, \mathrm{mEq} / \mathrm{L}$} \\
\hline Median [IQR] & 101 [98-104] \\
\hline$<98$, no./total no. $(\%)$ & $47 / 243(19 \%)$ \\
\hline$>107$, no./total no. $(\%)$ & $18 / 243(7 \%)$ \\
\hline
\end{tabular}

$* \mathrm{SpO}_{2}$ values are reported only in cases with $\mathrm{FiO}_{2}=0.21$. **Including coronary heart disease, cerebrovascular disease, heart failure, and peripheral vascular disease. $* * *$ Defined as $\mathrm{BMI} \geq 30$. Data are reported as median [IQR] (continuous/numerical variables) or number (\%) (binary variables). $A R D S$, acute respiratory distress syndrome; $E D$, emergency department; COVID-19, coronavirus disease 2019; RT-PCR, real-time reverse transcriptase-polymerase chain reaction; $C O P D$, chronic obstructive pulmonary disease; $\mathrm{PaO}_{2} / \mathrm{FiO}_{2}$ ratio, ratio of partial pressure of oxygen to fraction of inspired oxygen; $H B$, hemoglobin; $W B C$, white blood cells; PLT, platelets; INR, international normalized ratio; $a P T T$ ratio, activated partial thromboplastin time ratio; $A S T$, aspartate transaminase; $A L T$, alanine transaminase; $L D H$, lactate dehydrogenase; $C R P$, C-reactive protein; $\mathrm{SpO}_{2}$, oxygen saturation; $\mathrm{FiO}_{2}$, fraction of inspired oxygen; $I Q R$, interquartile range

first positive nasopharyngeal swab and those who became positive afterward.

The inter-rater agreement of CXR findings was almost perfect for the assessment of type of parenchymal opacity $(\kappa=0.90$; 95\% CI: $0.85,0.95)$, Brixia score (ICC $=0.91$; 95\% CI: $0.89,0.93)$, and percentage of lung involvement $(\mathrm{ICC}=0.95 ; 95 \%$ CI: 0.93, 0.96) [19] (Fig. 1). Notably, AP images showed an overall better inter-rater agreement than PA (Supplementary Material, Table S1). GGO admixed with consolidation was the most common finding (235/340, 69\%), followed by GGO (96/340, 28\%) (Fig. 2). Parenchymal opacities most frequently showed neither a peripheral nor a central distribution (219 out of 334 with parenchymal opacities, $65 \%$ ) or were peripherally located $(99 / 334,30 \%)$. Bilateral lung involvement was found in 312 cases $(93 \%)$ (Table 2). Patients with severe CXR findings more frequently suffered from dyspnea and were more likely to have laboratory abnormalities, including lower $\mathrm{SpO}_{2}$ and $\mathrm{PaO}_{2} / \mathrm{FiO}_{2}$ ratio values and raised inflammatory 
Fig. 1 Correlation and agreement between chest X-ray findings obtained by two independent reviewers in 340 patients with confirmed COVID-19.

Correlation and Bland-Altman plots show the agreement in Brixia score (a, b) and percentage of lung involvement (c, d) between the reference reviewer (reviewer 1, a thoracic radiologist with 5 years of experience) and reviewer 2 (a fourth-year radiology resident). In correlation plots, the dashed line denotes the line of perfect concordance, while the solid line denotes the reduced major axis. In Bland-Altman plots, the solid line denotes mean difference, while dashed lines denote mean difference \pm 2 standard deviations a
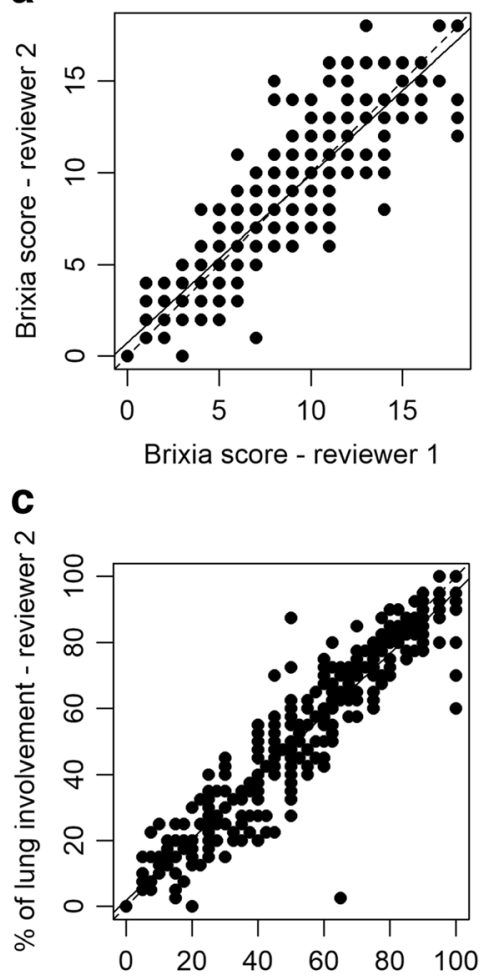

$\%$ of lung involvement - reviewer 1 b

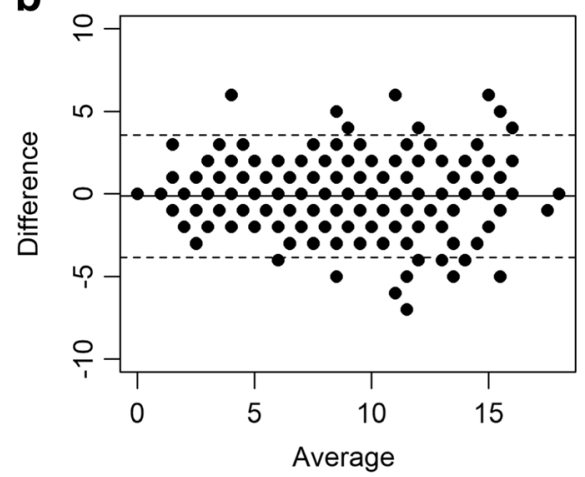

d

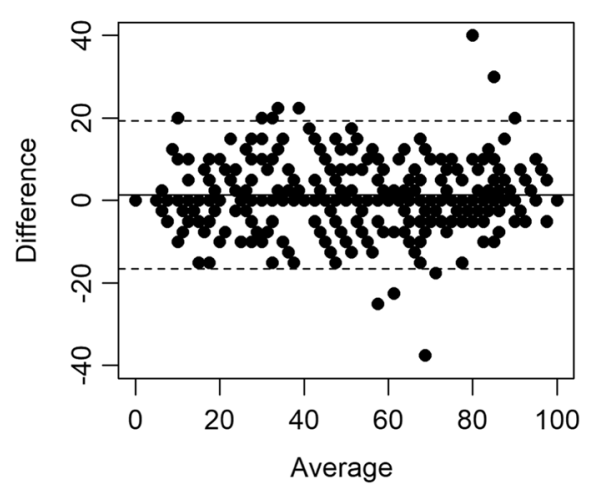

markers, liver enzymes, and creatinine levels (Supplementary Material, Table S2).

The main patients' outcomes are listed in Table 3. Median observation time was 63 days (IQR $=8-67)$. The two most frequent respiratory supports employed were oxygen mask $(144 / 340,42 \%)$ and continuous positive airway pressure/ noninvasive mechanical ventilation $(105 / 340,31 \%)$. Death occurred in $37 \%$ of cases $(125 / 340$, median age of 76$)$. A total

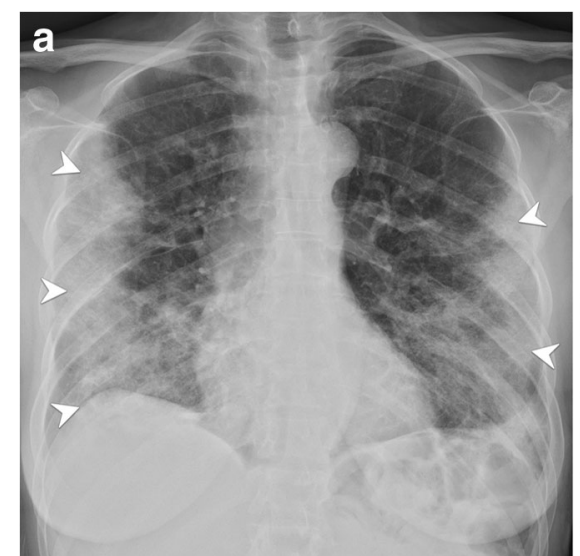

Fig. 2 Chest X-ray (CXR) findings at the emergency department presentation in two patients with confirmed COVID-19 and opposite outcomes. a CXR shows bilateral, mostly peripheral, ground-glass opacities (GGOs) admixed with consolidation (consolidationpredominant) (arrowheads). Reviewer 1 assigned a Brixia score of 14 and a percentage of lung involvement of $60 \%$. Reviewer 2 assigned a Brixia score of 15 and a percentage of lung involvement of $50 \%$. This patient had a prolonged stay in the intensive care unit and died 11 days

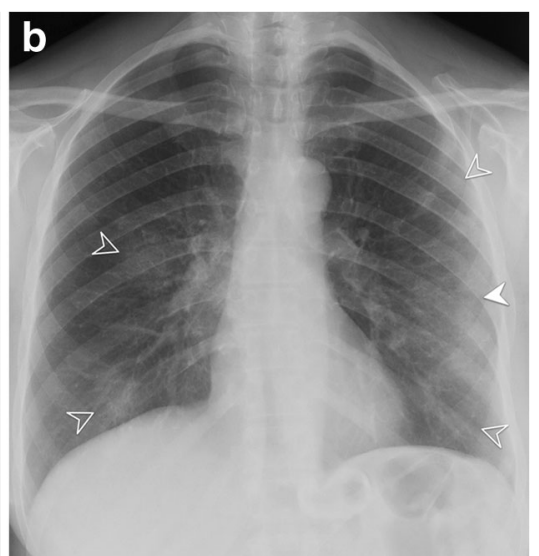

after presenting to the emergency department. b CXR shows bilateral GGOs, either pure (empty arrowheads) or admixed with consolidation (GGO-predominant) (solid arrowhead). Reviewer 1 assigned a Brixia score of 6 and a percentage of lung involvement of $30 \%$. Reviewer 2 assigned a Brixia score of 5 and a percentage of lung involvement of $25 \%$. This patient was discharged from the emergency department after a shortterm observation with home care and isolation precautions and was alive at the end of the study period 
Table 2 Chest X-ray analysis results obtained by two independent reviewers (reviewer 1 , a thoracic radiologist with 5 years of experience; reviewer 2, a fourth-year radiology resident) in 340 patients with confirmed COVID-19

\begin{tabular}{|c|c|c|c|c|}
\hline & Reviewer 1 & Reviewer 2 & Inter-rater agreement & $p$ \\
\hline Normal CXR & $6 / 340(2 \%)$ & $7 / 340(2 \%)$ & $\kappa=0.92[0.77-1.00]$ & $<0.001$ \\
\hline \multicolumn{3}{|l|}{ Type of parenchymal opacity } & \multirow[t]{5}{*}{$\kappa=0.90[0.85-0.95]$} & \multirow[t]{5}{*}{$<0.001$} \\
\hline GGO & $96 / 340(28 \%)$ & $100 / 340(29 \%)$ & & \\
\hline Consolidation & $3 / 340(1 \%)$ & $3 / 340(1 \%)$ & & \\
\hline GGO and consolidation & $235 / 340(69 \%)$ & $230 / 340(68 \%)$ & & \\
\hline None & $6 / 340(2 \%)$ & $7 / 340(2 \%)$ & & \\
\hline No. lung zones involved & $4[3-6]$ & $4[4-6]$ & $\mathrm{ICC}=0.86[0.83-0.88]$ & $<0.001$ \\
\hline$\geq 2$ lung zones involved & $321 / 340(94 \%)$ & $323 / 340(95 \%)$ & $\kappa=0.71[0.53-0.88]$ & $<0.001$ \\
\hline \multicolumn{3}{|l|}{ Distribution 1} & \multirow[t]{4}{*}{$\kappa=0.78[0.69-0.86]$} & \multirow[t]{4}{*}{$<0.001$} \\
\hline Central & $16 / 334(5 \%)$ & $8 / 333(3 \%)$ & & \\
\hline Peripheral & 99/334 (30\%) & 91/333 (27\%) & & \\
\hline Neither & $219 / 334(65 \%)$ & $234 / 333(70 \%)$ & & \\
\hline \multicolumn{3}{|l|}{ Distribution 2} & \multirow[t]{5}{*}{$\kappa=0.80[0.72-0.87]$} & \multirow[t]{5}{*}{$<0.001$} \\
\hline Superior & $3 / 334(1 \%)$ & $2 / 333(1 \%)$ & & \\
\hline Medium & $30 / 334(9 \%)$ & $22 / 333(6 \%)$ & & \\
\hline Inferior & $75 / 334(22 \%)$ & $69 / 333(21 \%)$ & & \\
\hline None & $226 / 334(68 \%)$ & $240 / 333(72 \%)$ & & \\
\hline \multicolumn{5}{|l|}{ Distribution 3} \\
\hline Bilateral & $312 / 334(93 \%)$ & $314 / 333(94 \%)$ & $\kappa=0.71[0.55-0.87]$ & $<0.001$ \\
\hline Pleural effusion & $53 / 340(16 \%)$ & $38 / 340(11 \%)$ & $\kappa=0.79[0.69-0.88]$ & $<0.001$ \\
\hline Nodules & $3 / 340(1 \%)$ & $1 / 340(0.3 \%)$ & $\kappa=0.50[-0.10$ to 1.00$]$ & 0.104 \\
\hline Brixia score [18] & $7[4-11]$ & $7[4-11]$ & $\mathrm{ICC}=0.91[0.89-0.93]$ & $<0.001$ \\
\hline$\%$ of lung involvement & $55[30-76]$ & 54 [28-75] & ICC $=0.95[0.93-0.96]$ & $<0.001$ \\
\hline
\end{tabular}

The frequency of individual CXR features is reported as number of positive cases or percent distribution. The Brixia score and percentage of lung involvement are shown in percent terms and median [IQR], respectively. Inter-rater agreement is shown as weighted Cohen's kappa (individual CXR features), or intraclass correlation coefficient (no. of lung zones involved, Brixia score, and percentage of lung involvement), with pertinent 95\% CI. $C X R$, chest $\mathrm{X}$-ray; $G G O$, ground-glass opacity; $I Q R$, interquartile range
Table 3 Outcomes of 340 patients with confirmed COVID-19. Followup information are reported as of May 12, 2020

\begin{tabular}{lc}
\hline Observation time, days & $63[8-67]$ \\
Length of hospitalization*, days & $7[4-14]$ \\
Respiratory support** & \\
None & $35 / 340(10 \%)$ \\
OM & $144 / 340(42 \%)$ \\
CPAP/NIV & $105 / 340(31 \%)$ \\
IV & $56 / 340(17 \%)$ \\
Death, no./total no. & $125 / 340(37 \%)$ \\
Time to death, days & $6[3-10]$ \\
ICU, no./total no. & $58 / 340(17 \%)$ \\
Age, year & $60[52-66]$ \\
Gender, F & $14 / 58(24 \%)$ \\
PEEP, cm $\mathrm{H}_{2} \mathrm{O} * * *$ & $16.5[15-18]$ \\
Prone position, no./total no. $(\%)$ & $26 / 41(63 \%)$ \\
ECMO, no./total no. $(\%)$ & $2 / 48(4 \%)$ \\
Deaths, no./total no. $(\%)$ & $22 / 58(38 \%)$
\end{tabular}

$*_{n}=33$ and $* * * n=12$ data missing due to patients' transfer to other hospital. **Refers to the most invasive respiratory support employed during observation time. Data are reported as median [IQR] (continuous/numerical variables) or number (\%) (binary variables). $O M$, oxygen mask; $C P A P / N I V$, continuous positive airway pressure/noninvasive mechanical ventilation; $I V$, invasive mechanical ventilation; $I C U$, intensive care unit; $P E E P$, positive end-expiratory pressure; $E C M O$, extracorporeal membrane oxygenation; $I Q R$, interquartile range of 58 patients ( $17 \%$, median age of 60 years) were admitted to ICU, among which 22 died (38\%, median age of 66).

Deceased patients were significantly older and had a higher number of comorbidities, significantly lower $\mathrm{SpO}_{2}$ and $\mathrm{PaO}_{2} / \mathrm{FiO}_{2}$ ratio values, and more severe CXR findings at ED admission than patients who survived $(p<0.001$ in all cases; Table 4). Significant differences in survival curves between age classes, $\mathrm{PaO}_{2} / \mathrm{FiO}_{2}$ ratio values, and several CXR findings (Brixia score, number of lung zones involved, and percentage of lung involvement) $(p<0.001$ in all cases) were found (Fig. 3).

On regression model analysis, the Brixia score (OR: $1.19 ; 95 \%$ CI: $1.06,1.34 ; p=0.003$ ), age (OR: 1.16; 95\% CI: $1.11,1.22 ; p<0.001), \mathrm{PaO}_{2} / \mathrm{FiO}_{2}$ ratio (OR: 0.99 ; 95\% CI: $0.98,1 ; p=0.002)$, and cardiovascular diseases (OR: 3.21; 95\% CI: 1.28, 8.39; $p=0.014$ ) significantly predicted death. Percentage of lung involvement (OR: 1.02; 95\% CI: 1.01, $1.03 ; p=0.001), \mathrm{SpO}_{2}$ (OR: 0.96; 95\% CI: 0.92, 0.99; $p=0.008$ ), $\mathrm{PaO}_{2} / \mathrm{FiO}_{2}$ ratio (OR: $0.99 ; 95 \% \mathrm{CI}: 0.99,1.00 ; p<0.001$ ), and rheumatic pathologies (OR: 3.22; 95\% CI: 1.05, 9.89; $p=0.041)$ predicted the need for ventilatory support (Table 5). The distribution of age, $\mathrm{PaO}_{2} / \mathrm{FiO}_{2}$ ratio, Brixia score, number of lung zones involved, and percentage of lung involvement by respiratory support employed is shown in Fig. 4. 
Table 4 Demographic, clinical, chest X-ray, and laboratory data of 340 patients with confirmed COVID-19 at ED presentation divided in groups based on their clinical outcome: deceased or survived

\begin{tabular}{|c|c|c|c|}
\hline & Deceased & Survived & $p$ \\
\hline No. & 125 & 215 & \\
\hline \multicolumn{4}{|l|}{ Age } \\
\hline Median [IQR], year & $76[70-82]$ & $61[54-70]$ & $<0.001$ \\
\hline \multicolumn{4}{|l|}{ Distribution, no./total no. (\%) } \\
\hline $18-59$ year & $6 / 125(5 \%)$ & $95 / 215(44 \%)$ & \\
\hline $60-69$ year & $23 / 125(18 \%)$ & $63 / 215(29 \%)$ & \\
\hline $70-79$ year & $54 / 125(43 \%)$ & $42 / 215(20 \%)$ & \\
\hline$\geq 80$ year & $42 / 125(34 \%)$ & $15 / 215(7 \%)$ & \\
\hline Gender, F, no./total no. $(\%)$ & $25 / 125(20 \%)$ & $63 / 215(29 \%)$ & 0.078 \\
\hline Smoking history, no./total no. (\%) & & & 0.135 \\
\hline Never smoked & $36 / 61(59 \%)$ & $66 / 104(63 \%)$ & \\
\hline Former smoker & $24 / 61(39 \%)$ & $30 / 104(29 \%)$ & \\
\hline Current smoker & $1 / 61(2 \%)$ & $8 / 104(8 \%)$ & \\
\hline \multicolumn{4}{|l|}{ Comorbidities, no./total no. (\%) } \\
\hline Any & 73/84 (87\%) & $94 / 131(72 \%)$ & 0.015 \\
\hline$>2$ & $33 / 84(39 \%)$ & $24 / 131(18 \%)$ & $<0.001$ \\
\hline Arterial hypertension & $78 / 125(62 \%)$ & $84 / 215(39 \%)$ & $<0.001$ \\
\hline Cardiovascular disease ${ }^{* *}$ & $52 / 125(42 \%)$ & $34 / 215(16 \%)$ & $<0.001$ \\
\hline Obesity*** & $21 / 84(25 \%)$ & $29 / 131(22 \%)$ & 0.749 \\
\hline Diabetes & $32 / 125(26 \%)$ & $22 / 215(10 \%)$ & $<0.001$ \\
\hline COPD & $11 / 125(9 \%)$ & $11 / 215(5 \%)$ & 0.270 \\
\hline Chronic renal failure & $8 / 125(6 \%)$ & $4 / 215(2 \%)$ & 0.060 \\
\hline Neoplasia (active history) & $15 / 125(12 \%)$ & $11 / 215(5 \%)$ & 0.037 \\
\hline \multicolumn{4}{|l|}{ CXR findings } \\
\hline Brixia score [18] & $10[7-14]$ & $6[4-10]$ & $<0.001$ \\
\hline$\%$ of lung involvement & $70[50-85]$ & $45[23-66]$ & $<0.001$ \\
\hline Type of parenchymal opacity & & & $<0.001$ \\
\hline GGO & $13 / 125(11 \%)$ & $83 / 215(39 \%)$ & \\
\hline Consolidation & $3 / 125(2 \%)$ & $0 / 215(0 \%)$ & \\
\hline GGO and consolidation & $109 / 125(87 \%)$ & $126 / 215(58 \%)$ & \\
\hline None & $0 / 125(0 \%)$ & $6 / 215(3 \%)$ & \\
\hline No. lung zones involved & $5[4-6]$ & $4[3-6]$ & $<0.001$ \\
\hline Bilateral parenchymal opacities & $121 / 125(97 \%)$ & $191 / 209(91 \%)$ & 0.089 \\
\hline Duration of symptoms, no. with data & 119 & 213 & 0.018 \\
\hline Median [IQR], days & $6[4-9]$ & $7[5-10]$ & \\
\hline \multicolumn{4}{|l|}{ Laboratory data at ED presentation } \\
\hline $\mathrm{SpO}_{2} *$, no. with data & 90 & 187 & $<0.001$ \\
\hline Median [IQR], \% & 86 [77-89] & 92 [89-95] & \\
\hline $\mathrm{PaO}_{2} / \mathrm{FiO}_{2}$ ratio, no. with data & 102 & 156 & \\
\hline Median [IQR] & $179[97-241]$ & $262[190-298]$ & $<0.001$ \\
\hline \multicolumn{4}{|l|}{ Distribution, no./total no. } \\
\hline$<100$, severe ARDS & $26 / 102(26 \%)$ & $15 / 156(10 \%)$ & \\
\hline 100-200, moderate ARDS & $31 / 102(30 \%)$ & $29 / 156(18 \%)$ & \\
\hline 200-300, mild ARDS & $40 / 102(39 \%)$ & $73 / 156(47 \%)$ & \\
\hline$>300$, normal & $5 / 102(5 \%)$ & $39 / 156(25 \%)$ & \\
\hline \multicolumn{4}{|l|}{$\mathrm{HB}, \mathrm{g} / \mathrm{dL}$} \\
\hline Median [IQR] & $13.4[12.1-14.7]$ & $13.9[12.6-15.0]$ & 0.033 \\
\hline$<14$, no./total no. $(\%)$ & $73 / 125(58 \%)$ & $109 / 213(51 \%)$ & \\
\hline$>17$, no./total no. $(\%)$ & $4 / 125(3 \%)$ & $6 / 213(3 \%)$ & \\
\hline \multicolumn{4}{|l|}{$\mathrm{WBC}, / \mathrm{mm}^{3}$} \\
\hline Median [IQR] & $7340[5050-9820]$ & $6230[4770-8420]$ & 0.061 \\
\hline$<4000$, no./total no. $(\%)$ & $17 / 125(14 \%)$ & $28 / 213(13 \%)$ & \\
\hline$>10000$, no./total no. $(\%)$ & $29 / 125(23 \%)$ & $37 / 213(17 \%)$ & \\
\hline \multicolumn{4}{|l|}{ Neutrophils } \\
\hline Median [IQR] (WBC \%) & $83[73-88]$ & 77 [71-83] & $<0.001$ \\
\hline Median $[\mathrm{IQR}]\left(/ \mathrm{mm}^{3}\right)$ & 5324 [3741-8421] & 4627 [3351-6755] & 0.058 \\
\hline$<2000$, no./total no. $(\%)$ & $5 / 111(4 \%)$ & $11 / 176(6 \%)$ & \\
\hline$>6700$, no./total no. $(\%)$ & $44 / 111(40 \%)$ & $45 / 176(26 \%)$ & \\
\hline \multicolumn{4}{|l|}{ Lymphocytes } \\
\hline Median [IQR] (WBC \%) & $9[7-14]$ & $15[10-19]$ & $<0.001$ \\
\hline Median $[\mathrm{IQR}]\left(/ \mathrm{mm}^{3}\right)$ & $662[546-886]$ & 928 [660-1236] & $<0.001$ \\
\hline$<1000$, no./total no. $(\%)$ & $67 / 82(82 \%)$ & $74 / 108(59 \%)$ & \\
\hline \multicolumn{4}{|l|}{ Monocytes } \\
\hline Median [IQR] (WBC \%) & $5[3-7]$ & $6[4-8]$ & 0.007 \\
\hline
\end{tabular}


Table 4 (continued)

\begin{tabular}{|c|c|c|c|}
\hline & Deceased & Survived & $p$ \\
\hline Median [IQR] $\left(/ \mathrm{mm}^{3}\right)$ & $330[224-512]$ & 385 [256-557] & 0.179 \\
\hline$<250$, no./total no. $(\%)$ & $24 / 82(29 \%)$ & $26 / 108(24 \%)$ & \\
\hline$>800$, no./total no. $(\%)$ & $7 / 82(9 \%)$ & $9 / 108(8 \%)$ & \\
\hline \multicolumn{4}{|l|}{ INR } \\
\hline Median $[\mathrm{IQR}]$ & $1.10[1.05-1.18]$ & $1.05[1.01-1.11]$ & $<0.001$ \\
\hline$>1.25$, no. $/$ total no. $(\%)$ & 19/108 (18\%) & 15/171 (9\%) & \\
\hline \multicolumn{4}{|l|}{ aPTT ratio } \\
\hline Median [IQR] & $1.21[1.11-1.33]$ & $1.10[1.00-1.20]$ & $<0.001$ \\
\hline$>1.25$, no./total no. $(\%)$ & $51 / 118(43 \%)$ & $35 / 188(19 \%)$ & \\
\hline \multicolumn{4}{|l|}{ Creatinine, $\mathrm{mg} / \mathrm{dL}$} \\
\hline Median $[\mathrm{IQR}]$ & $1.12[0.81-1.50]$ & $0.90[0.75-1.04]$ & $<0.001$ \\
\hline$>1.30$, no./total no. $(\%)$ & $44 / 125(35 \%)$ & $32 / 212(15 \%)$ & \\
\hline \multicolumn{4}{|l|}{ Urea, $\mathrm{mg} / \mathrm{dL}$} \\
\hline Median [IQR] & $58[43-88]$ & $39[30-48]$ & $<0.001$ \\
\hline$>50$, no./total no. $(\%)$ & $70 / 110(64 \%)$ & $39 / 175(22 \%)$ & \\
\hline \multicolumn{4}{|l|}{$\mathrm{LDH}, \mathrm{U} / \mathrm{L}$} \\
\hline Median [IQR] & 485 [376-633] & 372 [294-464] & $<0.001$ \\
\hline$\geq 250$, no./total no. $(\%)$ & $107 / 113(95 \%)$ & $171 / 193(89 \%)$ & \\
\hline \multicolumn{4}{|l|}{$\mathrm{CRP}, \mathrm{mg} / \mathrm{dL}$} \\
\hline Median [IQR] & 15 [10-22] & $10[4-15]$ & $<0.001$ \\
\hline$\geq 1$, no./total no. $(\%)$ & $122 / 123(99 \%)$ & $201 / 210(96 \%)$ & \\
\hline \multicolumn{4}{|l|}{$\mathrm{K}, \mathrm{mEq} / \mathrm{L}$} \\
\hline Median [IQR] & $4.0[3.7-4.4]$ & $3.9[3.6-4.2]$ & 0.008 \\
\hline$<3.5$, no./total no. $(\%)$ & $15 / 122(12 \%)$ & $40 / 209(19 \%)$ & \\
\hline$>5$, no. $/$ total no. $(\%)$ & $13 / 122(11 \%)$ & $6 / 209(3 \%)$ & \\
\hline
\end{tabular}

$* \mathrm{SpO}_{2}$ reported only in cases with $\mathrm{FiO}_{2}=0.21$. $* *$ Including coronary heart disease, cerebrovascular disease, heart failure, and peripheral vascular disease. $* * *$ Defined as $\mathrm{BMI} \geq 30$. Data are reported as median $[\mathrm{IQR}]$ (continuous/numerical variables) or number (\%) (binary variables). $P$ values are computed Mann-Whitney test (continuous variables), or chi-squared test (binary and categorical variables). COVID-19, coronavirus disease of 2019; $\mathrm{COPD}$, chronic obstructive pulmonary disease; $\mathrm{PaO}_{2} / \mathrm{FiO}_{2}$ ratio, ratio of partial pressure of oxygen to fraction of inspired oxygen; $\mathrm{HB}$, hemoglobin; $W B C$, white blood cells; $I N R$, international normalized ratio; $a P T T$, activated partial thromboplastin time; $L D H$, lactate dehydrogenase; $C R P, \mathrm{C}$-reactive protein; $\mathrm{SpO}_{2}$, oxygen saturation; $\mathrm{FiO}_{2}$, fraction of inspired oxygen; $E D$, emergency department; $G G O$, ground-glass opacity; $A R D S$, acute respiratory distress syndrome; $I Q R$, interquartile range

\section{Discussion}

The ongoing COVID-19 pandemic has highlighted the need for prompt diagnostic and prognostic strategies to optimize patient management, especially when the availability of critical care resources is limited or overwhelmed. In the present study, the vast majority of COVID-19 patients $(334 / 340,99 \%)$ had signs of pneumonia on CXR, even those who tested negative at initial RTPCR. Presence, distribution, and type of parenchymal opacity (i.e., GGO, consolidation, or both), as well as the Brixia score and the percentage of lung involvement, were consistently assessed by two independent reviewers with different levels of expertise. We found that a higher Brixia score, increasing age, underlying cardiovascular diseases, and lower $\mathrm{PaO}_{2} / \mathrm{FiO}_{2}$ ratio values were significant predictors of death. The main predictors of the need for ventilatory support were found to be a higher percentage of lung involvement on CXR, the presence of rheumatic pathologies, and lower $\mathrm{SpO}_{2}$ and $\mathrm{PaO}_{2} / \mathrm{FiO}_{2}$ ratio values.
A few studies have examined the value of CXR to predict COVID-19 outcomes. In early May 2020, Borghesi et al introduced the Brixia score, an experimental CXR scoring system for quantifying lung abnormalities in COVID-19 pneumonia [18]. High Brixia score values have been found to predict in-hospital mortality for COVID-19 [15]. Also, Toussie et al found that a lung zone severity score on the initial CXR was associated with the need for intubation in COVID-19 patients aged 21-50 years [16]. No studies have investigated the value of initial CXR to predict mortality in COVID-19 patients so far. In the present study, we first demonstrated that Brixia score on initial CXR is predictive of fatal outcome (based on in-hospital and out-of-hospital deaths) in COVID-19 patients. Moreover, in keeping with Toussie et al [16], we found the percentage of lung involvement to be a predictor of the need for ventilatory support. Although the Brixia score and the percentage of lung involvement were significant predictors of both mortality and ventilatory support in the univariate analysis, only one of them remained significant in each outcome's 
Fig. 3 Survival curves related to 340 patients with confirmed COVID-19, grouped by demographic variables (a: age, b: sex), $\mathrm{PaO}_{2} / \mathrm{FiO}_{2}$ ratio (c), and chest $\mathrm{X}$-ray findings at presentation to the emergency department (d: Brixia score, e: number of lung zones involved, $\mathbf{f}$ : percentage of lung involvement), over a median of 63 days observation time. Shadows denote $95 \%$ confidence intervals, while $p$ denotes the significance of the difference between strata at log-rank test. $\mathrm{PaO}_{2} / \mathrm{FiO}_{2}$ ratio, ratio of partial pressure of oxygen to fraction of inspired oxygen
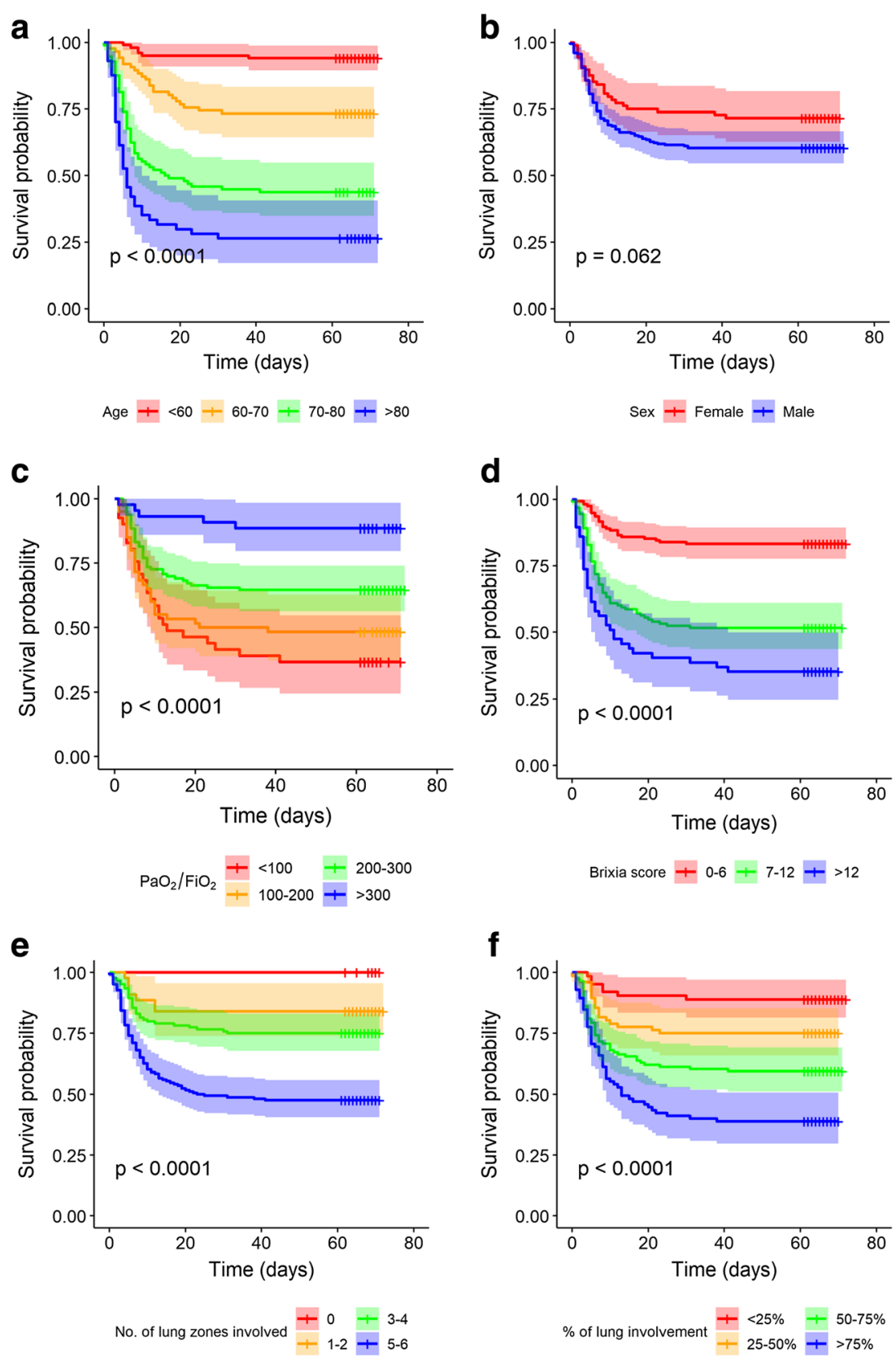

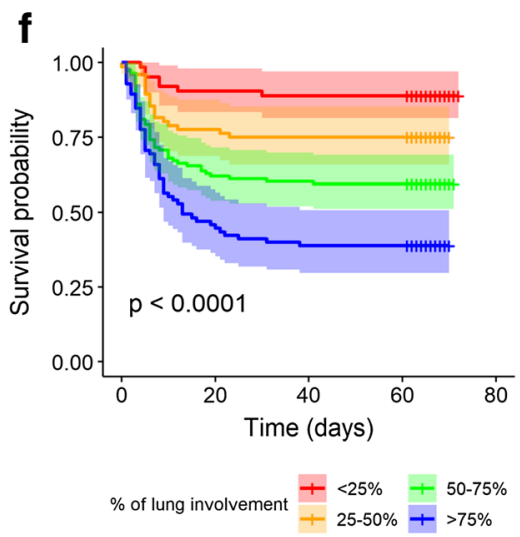

multivariate model because the two scores provide partially overlapping information. The number of lung zones involved and type of parenchymal opacities were also significantly different between survivors and deceased patients, the latter more frequently presenting with a greater degree of lung involvement and consolidation. Remarkably, the overall inter-rater agreement was better for AP images, where the higher disease severity may have led to relatively obvious CXR findings.
Our study population was mainly composed of patients presenting in a relatively advanced stage of the disease, with a median number of days from symptom onset to ED presentation of 7 . The proportion of normal CXRs $(6 / 340,2 \%)$ was, therefore, significantly lower than those reported in previous studies where patients presented earlier in the course of their disease $[11,16,20]$. In accordance with reports showing the highest radiological severity of the disease approximately 6-11 days after the onset 
Table 5 Demographic, clinical, laboratory, and chest X-ray data that demonstrated predictive value for death and the most invasive respiratory support employed (none, oxygen mask, continuous positive airway pressure/noninvasive ventilation or invasive ventilation) in 340 patients with confirmed COVID-19

\begin{tabular}{|c|c|c|c|c|}
\hline & Predictors & Odds ratio & $95 \% \mathrm{CI}$ & $p$ \\
\hline \multirow[t]{5}{*}{ Death } & Age, years & 1.16 & {$[1.11-1.22]$} & $<0.001$ \\
\hline & $\mathrm{SpO}_{2}, \% *$ & 0.96 & {$[0.92-1.01]$} & 0.115 \\
\hline & $\mathrm{PaO}_{2} / \mathrm{FiO}_{2}$ ratio* & 0.99 & {$[0.98-1.00]$} & 0.002 \\
\hline & Cardiovascular disease ${ }^{* *}$ & 3.21 & [1.28-8.39] & 0.014 \\
\hline & Brixia score [18] & 1.19 & {$[1.06-1.34]$} & 0.003 \\
\hline \multirow[t]{5}{*}{ Respiratory support } & Age, years & 0.96 & {$[0.94-0.98]$} & 0.001 \\
\hline & $\mathrm{SpO}_{2}, \% *$ & 0.96 & {$[0.92-0.99]$} & 0.008 \\
\hline & $\mathrm{PaO}_{2} / \mathrm{FiO}_{2}$ ratio $*$ & 0.99 & {$[0.99-1.00]$} & $<0.001$ \\
\hline & Rheumatic pathology & 3.22 & [1.05-9.89] & 0.041 \\
\hline & $\%$ of lung involvement & 1.02 & [1.01-1.03] & 0.001 \\
\hline
\end{tabular}

*Within $24 \mathrm{~h}$ of ED presentation. **Including coronary heart disease, cerebrovascular disease, heart failure, and peripheral vascular disease. $\mathrm{SpO}_{2}$ reported only in cases with $\mathrm{FiO}_{2}=0.21$. Odds ratios, $95 \% \mathrm{CI}$, and $p$ values were computed by logistic (death) and ordinal logistic (respiratory support) regression models, using stepwise model selection technique. Only patients with no missing data $(n=210)$ were included in the models. $E D$, emergency department; $\mathrm{PaO}_{2} / \mathrm{FiO}_{2}$ ratio, ratio of partial pressure of oxygen to fraction of inspired oxygen; $\mathrm{SpO}_{2}$, oxygen saturation

of symptoms [21, 22], we found CXR signs of advanced pneumonia in a high proportion of patients: GGO admixed with consolidation $(235 / 340,69 \%)$, bilateral parenchymal opacities $(312 / 334,93 \%)$, and a median percentage of lung involvement of 55. Pleural effusion was recorded in a higher percentage of patients $(53 / 340,16 \%)$ than that previously reported [11].

Unlike previous findings [23], we did not find any significant differences between patients with a positive initial RT-PCR result and those who became positive afterward, both presenting with a high prevalence of GGO admixed with consolidation ( $68 \%$ and $81 \%$, respectively). Moreover, all of the 27 patients who tested negative on initial RT-PCR had CXR findings suggestive of pneumonia, thus underlining the potential of CXR as a valuable complementary diagnostic tool in the first-line work-up of suspected COVID-19 patients.

In accordance with Du et al [24], our findings confirm that older age and cardiovascular diseases predict fatal outcome in COVID-19 patients. As expected, a greater number of comorbidities, hypertension, and diabetes were also found to be associated with death. However, in accordance with previous findings [25], these parameters did not remain significant predictors of mortality in multivariate analysis. In line with a recently published larger series, neither smoking nor obesity (defined as $\mathrm{BMI} \geq 30$ ) was found to be associated with death [26].

$\mathrm{PaO}_{2} / \mathrm{FiO}_{2}$ ratio was found to be a significant predictor of death and the need for ventilatory support, while $\mathrm{SpO}_{2}$ was a significant predictor of the need for ventilatory support only. $\mathrm{PaO}_{2} / \mathrm{FiO}_{2}$ ratio, as a surrogate of hypoxia, was previously found to be a predictor of death in other types of pneumonia [27] and to appear significantly lower in patients deceased of COVID-19 compared with those who survived [28]. In our cohort, most patients presented with a mild respiratory failure (median $\mathrm{PaO}_{2} / \mathrm{FiO}_{2}$ ratio $=238$ ), and $\mathrm{PaO}_{2} / \mathrm{FiO}_{2}$ ratio at $\mathrm{ED}$ presentation was significantly reduced in deceased patients compared with that in survivors (179 vs. 262, $p<0.001)$. Our results provide further evidence in support of the $\mathrm{PaO}_{2} / \mathrm{FiO}_{2}$ ratio as a critical parameter to assess disease severity in patients with severe respiratory symptoms due to COVID-19.

The "protective" role of increasing age against the need for ventilatory support found in the present study can be safely considered artifactual. Reasonably, this result has been influenced by the extraordinary distribution of limited healthcare resources, preferentially allocated to patients with a higher possibility of therapeutic success and life expectancy.

The present study has some limitations. First, in such an emergency, the completeness of data recorded was less than optimal. Moreover, our cohort attended the ED after several days from symptom onset and in a relatively advanced disease stage, thus making the generalizability of our results uncertain. Also, such an imbalance between clinical needs and availability of intensive care resources has reasonably led to discrepancies between disease severity or clinical outcomes and mode of respiratory support employed. Lastly, given the 
Fig. 4 Distribution of age (a), $\mathrm{PaO}_{2} / \mathrm{FiO}_{2}$ ratio (b), and $\mathrm{CXR}$ findings (c: Brixia score, $\mathbf{d}$ : percentage of lung involvement, e: number of lung zones involved) at presentation to the emergency department by the most invasive respiratory support employed in 340 patients with confirmed COVID-19. CXR, chest X-ray; ED, emergency department; $\mathrm{PaO}_{2} / \mathrm{FiO}_{2}$ ratio, ratio of partial pressure of oxygen to fraction of inspired oxygen; OM, oxygen mask; CPAP/NIV, continuous positive airway pressure/ noninvasive mechanical ventilation; IV, invasive mechanical ventilation
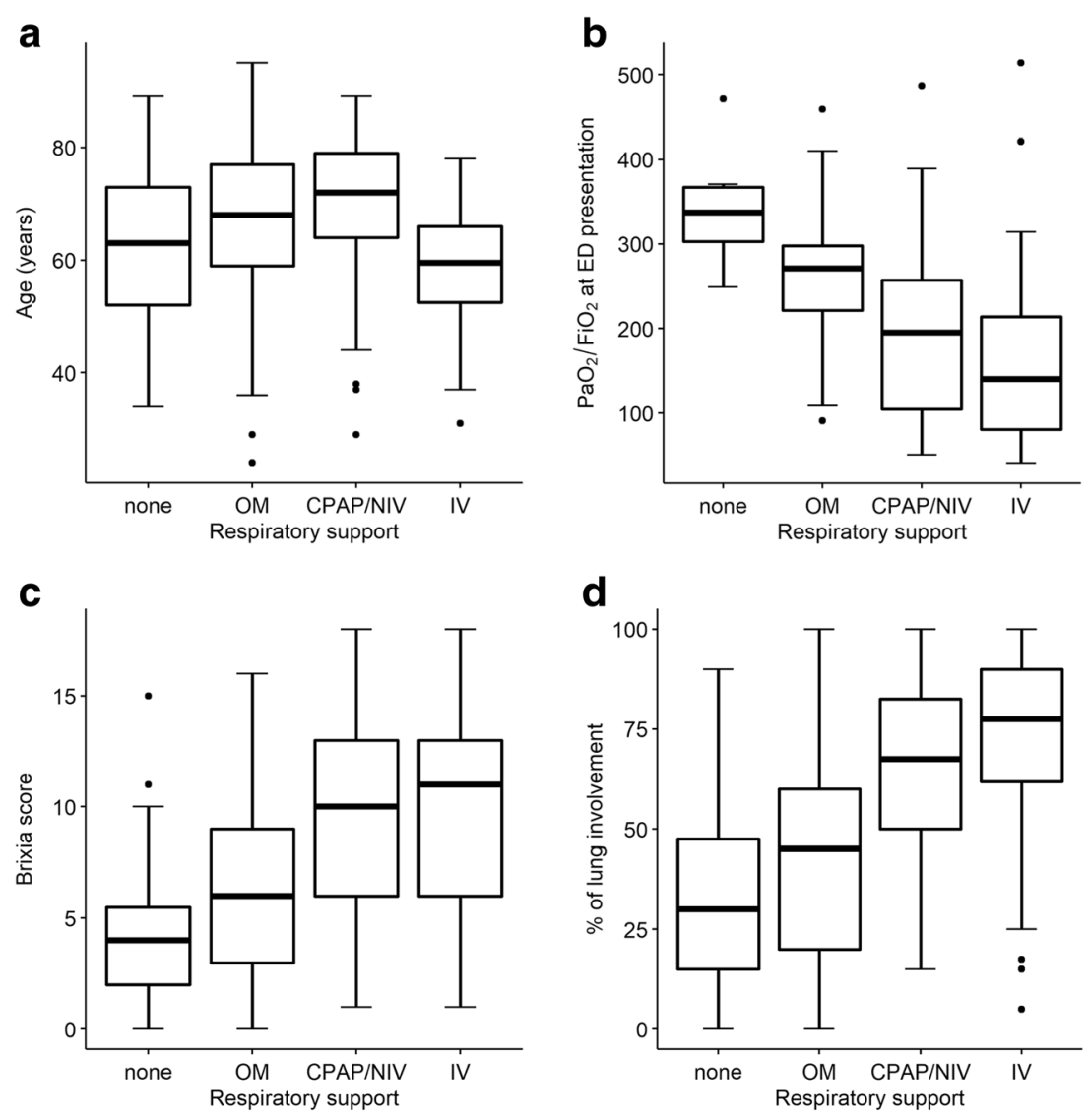

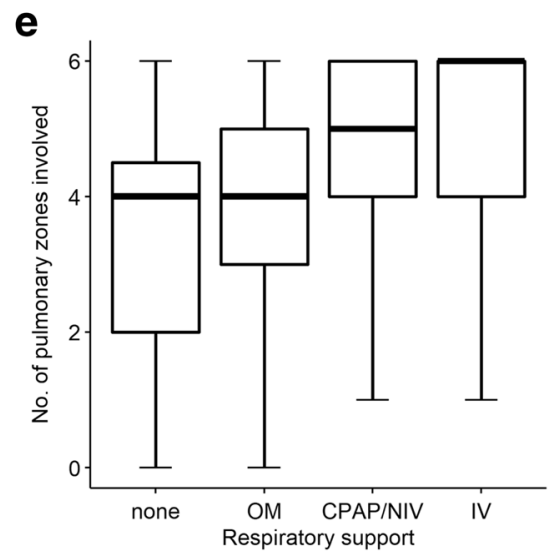

very low number of $\mathrm{CT}$ scans performed in our institution in this emergency situation and considering that most of them were not acquired at ED presentation but rather later in the disease course, a comparison between CT and CXR findings was not feasible.

In conclusion, CXR is a reproducible tool for assessing COVID-19. Along with patient history, $\mathrm{SpO}_{2}$, and $\mathrm{PaO}_{2} /$ $\mathrm{FiO}_{2}$ ratio values, $\mathrm{CXR}$ at ED presentation may help to identify patients at risk for death and ventilatory support, thus enabling to optimize clinical management in high-prevalence settings of the disease.

Funding Open access funding provided by Università degli Studi di Milano - Bicocca within the CRUI-CARE Agreement.

\section{Compliance with ethical standards}

Guarantor The scientific guarantor of this publication is Dr. Pietro Andrea Bonaffini. 
Conflict of interest The authors of this manuscript declare no relationships with any companies whose products or services may be related to the subject matter of the article.

Statistics and biometry One of the authors has significant statistical expertise.

Informed consent Written informed consent was waived by the Institutional Review Board.

Ethical approval Institutional Review Board approval was obtained.

\author{
Methodology \\ - retrospective \\ - observational \\ - performed at one institution
}

Open Access This article is licensed under a Creative Commons Attribution 4.0 International License, which permits use, sharing, adaptation, distribution and reproduction in any medium or format, as long as you give appropriate credit to the original author(s) and the source, provide a link to the Creative Commons licence, and indicate if changes were made. The images or other third party material in this article are included in the article's Creative Commons licence, unless indicated otherwise in a credit line to the material. If material is not included in the article's Creative Commons licence and your intended use is not permitted by statutory regulation or exceeds the permitted use, you will need to obtain permission directly from the copyright holder. To view a copy of this licence, visit http://creativecommons.org/licenses/by/4.0/.

\section{References}

1. Guan WJ, Ni ZY, Hu Y et al (2020) Clinical characteristics of coronavirus disease 2019 in China. N Engl J Med 382(18):17081720

2. Lomoro P, Verde F, Zerboni F et al (2020) COVID-19 pneumonia manifestations at the admission on chest ultrasound, radiographs, and CT: single-center study and comprehensive radiologic literature review. Eur J Radiol Open 7:100231

3. World Health Organization (2020) Clinical management of COVID-19: interim guidance, 27 May 2020 (No. WHO/2019$\mathrm{nCoV} /$ clinical/2020.5). World Health Organization

4. Wang W, Xu Y, Gao R et al (2020) Detection of SARS-CoV-2 in different types of clinical specimens. JAMA 323(18):1843-1844

5. Ai T, Yang Z, Hou H et al (2020) Correlation of chest CT and RTPCR testing in coronavirus disease 2019 (COVID-19) in China: a report of 1014 cases. Radiology 200642

6. Sverzellati N, Milanese G, Milone F, Balbi M, Ledda RE, Silva M (2020) Integrated radiologic algorithm for COVID-19 pandemic. J Thorac Imaging. https://doi.org/10.1097/RTI.0000000000000516

7. Rubin GD, Ryerson CJ, Haramati LB et al (2020) The role of chest imaging in patient management during the COVID-19 pandemic: a multinational consensus statement from the Fleischner Society. Chest. https://doi.org/10.1016/j.chest.2020.04.003

8. The BMJ (2020) Lessons from the frontline of the covid-19 outbreak. BMJ. Available via https://blogs.bmj.com/bmj/2020/03//20/ lessons-from-the-frontline-of-the-covid-19-outbreak/?utm campaign $=$ shareaholic\&utm medium $=$ twitter\&utm source $=$ socialnetwork. Accessed 22 Mar 2020
9. American College of Radiology (2020) ACR recommendations for the use of chest radiography and computed tomography (CT) for suspected COVID-19 infection. American College of Radiology. Available via https://www.acr.org/Advocacy-and-Economics/ ACR-Position-Statements/Recommendations-for-ChestRadiography-and-CT-for-Suspected-COVID19-Infection. Accessed 22 Mar 2020

10. Jacobi A, Chung M, Bernheim A, Eber C (2020) Portable chest Xray in coronavirus disease-19 (COVID-19): a pictorial review. Clin Imaging 64:35-42

11. Wong HYF, Lam HYS, Fong AH et al (2020) Frequency and distribution of chest radiographic findings in COVID-19 positive patients. Radiology 201160

12. Vancheri SG, Savietto G, Ballati F et al (2020) Radiographic findings in 240 patients with COVID-19 pneumonia: time-dependence after the onset of symptoms. Eur Radiol:1-9

13. Hui DS, Wong KT, Antonio GE et al (2004) Severe acute respiratory syndrome: correlation between clinical outcome and radiologic features. Radiology 233(2):579-585

14. Antonio GE, Wong KT, Tsui EL et al (2005) Chest radiograph scores as potential prognostic indicators in severe acute respiratory syndrome (SARS). AJR Am J Roentgenol 184(3):734-741

15. Borghesi A, Zigliani A, Golemi S et al (2020) Chest X-ray severity index as a predictor of in-hospital mortality in coronavirus disease 2019: a study of 302 patients from Italy. Int J Infect Dis 96:291-293

16. Toussie D, Voutsinas N, Finkelstein M et al (2020) Clinical and chest radiography features determine patient outcomes in young and middle age adults with COVID-19. Radiology 201754

17. Hansell DM, Bankier AA, MacMahon H, McLoud TC, Müller NL, Remy J (2008) Fleischner Society: glossary of terms for thoracic imaging. Radiology 246(3):697-722

18. Borghesi A, Maroldi R (2020) COVID-19 outbreak in Italy: experimental chest X-ray scoring system for quantifying and monitoring disease progression. Radiol Med 125(5):509-513

19. Landis JR, Koch GG (1977) The measurement of observer agreement for categorical data. Biometrics 33(1):159-174

20. Weinstock MB, Echenique A, Russell JW et al (2020) Chest x-ray findings in 636 ambulatory patients with COVID-19 presenting to an urgent care center: a normal chest $\mathrm{x}$-ray is no guarantee. J Urgent Care Med 14(7):13-18

21. Wang Y, Dong C, Hu Y et al (2020) Temporal changes of CT findings in 90 patients with COVID-19 pneumonia: a longitudinal study. Radiology 200843

22. Pan F, Ye T, Sun P et al (2020) Time course of lung changes on chest CT during recovery from 2019 novel coronavirus (COVID19) pneumonia. Radiology 295(3):715-721

23. Chen D, Jiang X, Hong Y et al (2020) Can chest CT features distinguish patients with negative from those with positive initial RT-PCR results for coronavirus disease (COVID-19)? AJR Am J Roentgenol: $1-5$

24. Du RH, Liang LR, Yang CQ et al (2020) Predictors of mortality for patients with COVID-19 pneumonia caused by SARS-CoV-2: a prospective cohort study. Eur Respir J 55(5):2000524

25. Wu C, Chen X, Cai Y et al (2020) Risk factors associated with acute respiratory distress syndrome and death in patients with coronavirus disease 2019 pneumonia in Wuhan, China. JAMA Intern Med e200994.

26. Petrilli CM, Jones SA, Yang J et al (2020) Factors associated with hospital admission and critical illness among 5279 people with coronavirus disease 2019 in New York City: prospective cohort study. BMJ 369:m1966 
27. Shi SJ, Li H, Liu M et al (2017) Mortality prediction to hospitalized patients with influenza pneumonia: $\mathrm{PO}_{2} / \mathrm{FiO}_{2}$ combined lymphocyte count is the answer. Clin Respir J 11(3):352-360

28. Yang X, Yu Y, Xu J et al (2020) Clinical course and outcomes of critically ill patients with SARS-CoV-2 pneumonia in Wuhan, China: a single-centered, retrospective, observational study.
Lancet Respir Med 8(5):475-481. https://doi.org/10.1016/S22132600(20)30079-5

Publisher's note Springer Nature remains neutral with regard to jurisdictional claims in published maps and institutional affiliations. 